\title{
STELLAR NEIGHBORHOODS IN POLYHEDRAL MANIFOLDS
}

\author{
RONALD H. ROSEN ${ }^{1}$
}

Introduction. It is the purpose of this paper to give some weaker analogues for polyhedral manifolds of well-known properties of combinatorial manifolds. This is now possible primarily because of the recent work of Mazur $[3 ; 4]$ and M. Brown [1]. A crucial issue in this area is the extent to which these analogues may be improved for arbitrary triangulated manifolds.

We shall prove a theorem which will be applied later on, after making some preliminary definitions. The join of two spaces $X$ and $Y$ is represented by $X \circ Y$. A map $f$ of $X \circ Y-Y$ on itself is called ray preserving if for each ray $p \circ q-q, p$ in $X, q$ in $Y, f(p \circ q-q)$ $\subseteq p \circ q-q$. A subset $K$ of a cone $X \circ p$ is called starlike if $p \in K$ and each segment $p \circ x, x \in X$, meets $K$ in a connected set; $p$ is the center of $K$. Consider $E^{n}$ to be the open cone over $S^{n-1}$ from the origin $p$, in the usual way. We coordinatize $E^{n}$ by $(x, t), x \in S^{n-1}, t$ a real number with $0 \leqq t<\infty ;\left(S^{n-1}, 0\right)=p$. Let $D^{n}$ be the unit $n$-ball $p \circ\left(S^{n-1}, 1\right)$.

THEOREM 1. Let $K$ be a compact starlike set with center $p$, lying in the interior of $D^{n}$. There is a ray preserving map $f$ of $E^{n}$ on itself such that $f(K)=p, f \mid E^{n}-K$ is one-to-one and $f \mid E^{n}-D^{n}$ is the identity.

Proof. Let $\rho$ be the usual euclidean metric for $E^{n}$. Choose $0<\epsilon<1$ so that $K \subseteq p \circ\left(S^{n-1}, \epsilon\right)=D_{\epsilon}$. First we define $f$ on $D_{\epsilon}$. If $z=(x, t) \in D_{\epsilon}$, $f(z)=(x, \rho(z, K))$. It is clear that $f$ is a ray preserving map and monotone nonincreasing with respect to the coordinate $t$, so $f\left(D_{\epsilon}\right) \subseteq D_{\epsilon}$; furthermore $f(K)=p$ and $f \mid D_{\epsilon}-K$ is one-to-one. To see the last property suppose $\left(x, t_{1}\right)$ and $\left(x, t_{2}\right)$ are points of $D_{e}-K$ with $t_{1}<t_{2}$. Let $y \in K$ so that $\rho\left(\left(x, t_{2}\right), y\right)=\rho\left(\left(x, t_{2}\right), K\right)$. Since $y \circ p \subseteq K$ and $\rho$ is the ordinary straight line distance, obviously $\rho\left(\left(x, t_{1}\right), y \circ p\right)$ $<\rho\left(\left(x, t_{2}\right), y\right)$ and accordingly $f\left(x, t_{1}\right) \neq f\left(x, t_{2}\right)$.

We next extend $f$ to the annulus $D^{n}-D_{\epsilon}$ so that $f$ is fixed on $\operatorname{Bd} D^{n}$. If $z=(1-\tau) \cdot(x, \epsilon)+\tau \cdot(x, 1), 0 \leqq \tau \leqq 1$ and $x \in S^{n-1}$, let $f(z)=(1-\tau)$ $\cdot f(x, \epsilon)+\tau \cdot(x, 1)$. Finally let $f$ be fixed outside of $D^{n}$. Now $f$ has the

Presented to the Society, October 2, 1961; received by the editors January 12, 1962.

${ }^{1}$ This research in its original form was supported by contract NSF G18869. Revisions including the addition of Theorem 9 were supported by contract Nonr $(G)$ 00098-62. 
desired properties and is also monotone nonincreasing in the $t$ coordinate. Clearly $f$ is realizable as the end of a ray preserving pseudo-isotopy starting at the identity, which is fixed outside of $D^{n}$ and is monotone nonincreasing in $t$.

Corollary. $f$ is a homeomorphism of $E^{n}-K$ onto $E^{n}-p$.

Theorem 2. Suppose $X$ is a compact metric finite dimensional space and $K$ is a compact starlike set in $X \circ p-X$. There is a ray preserving map $f$ of $X \circ p$ on itself so that $f(K)=p, f$ is a homeomorphism on $X \circ p-K$ and $f$ is fixed on a neighborhood of $X{ }^{2}$

Proof. Suppose $X$ is imbedded in $\left(S^{n-1}, 1\right) \subseteq E^{n}$. This is extended in the obvious way to an imbedding of $X \circ p$ in $D^{n}$. $K$ is now starlike in Int $D^{n}$ so we apply Theorem 1 here. Since the map we then get is ray preserving and maps $D^{n}$ on itself, clearly it maps $X \circ p$ on itself. The restriction of this map to $X \circ p$ satisfies the necessary conditions except possibly the last, however a slight alteration can be made in the proof of Theorem 1 so that the map constructed therein is also fixed on some neighborhood of $\mathrm{Bd} D^{n}$.

1. A theorem due to Mazur. In [3] Mazur indicated that Theorem 4 of this section would follow from the form of the Generalized Schoenflies Theorem later proved by M. Brown [1] and Morse [6]; he also gave a proof of Theorem 5 in [4]. In [7] we indicated a simpler proof of the latter, using Theorem 4 . For completeness we include our own proofs.

Theorem 3 (Moise-AleXANDer). Let $M$ be a compact Hausdorff space which is the union of two open sets, each of which is homeomorphic to $E^{n}$. Then $M$ is homeomorphic to $S^{n}$.

This was conjectured by Alexander for $n=3$ and proved by Moise in that case [5]. Many people, including the author, have independently noticed that the proof for general $n$ follows from [1] in a manner quite similar to Moise's proof.

Theorem 4 (MAzur). If $Y \circ p$ is locally $n$-euclidean at $p$, then the suspension $S(Y)$ is homeomorphic to $S^{n}$.

Proof. Consider $S(Y)$ as $Y \times[-1,1]$ with $Y_{-1}$ and $Y_{1}$ identified as distinct points $p^{\prime}$ and $p$, respectively. Suppose $U$ is a euclidean neighborhood of $p$ in $S(Y)$ so that $\bar{U}$ is compact. For some $t_{0}$, $-1<t_{0}<1, U\left(Y_{t}: t_{0} \leqq t \leqq 1\right) \subseteq U$. $Y_{t_{0}}$ is closed in $S(Y)$, so $Y$, hence

\footnotetext{
${ }^{2} \mathrm{~A}$ more general theorem can be proved but this is sufficient for our applications.
} 
$S(Y)$, is compact. Let $h$ be a homeomorphism of $S(Y)$ on itself, fixed on $Y_{t_{0}}$, which takes $U\left(Y_{t}: t_{0} \leqq t\right)$ onto $U\left(Y_{t}: t \leqq t_{0}\right)$. $U$ and $h(U)$ are two euclidean neighborhoods which cover $S(Y)$.

Corollary. $Y \circ p-Y$ is homeomorphic to $E^{n}$.

Proof. There is a simple homeomorphism of $Y \circ p-Y$ onto $\mathrm{U}\left(Y_{t}: 0<t\right)$ in $S(Y)$ and a homeomorphism of the second set onto $S(Y)-p^{\prime}$.

TheOREM 5 (MAZUR). Let $v$ be a vertex of a triangulated $n$-manifold $M$. The open star of $v$ in $M$ is homeomorphic to $E^{n}$.

Proof. Let $B$ be the link of $v$ in $M$. Since $B \circ v-B$ is a neighborhood of $v$ in $M$, by Theorem $4 S(B) \approx S^{n}$. (We use the notation $X \approx Y$ if $X$ and $Y$ are homeomorphic spaces.) Therefore by the corollary, $B \circ v-B \approx E^{n}$.

2. Some analogues to combinatorial theorems. M. Brown has called a subset of an $n$-manifold cellular if it is the intersection of a sequence $\left(C_{n}\right)$ of topological $n$-cells where $C_{n+1} \subseteq$ Int $C_{n}$. Let $Q$ be a subpolyhedron of a polyhedron $P$. The stellar neighborhood of $Q$ in $P$ is the union of all open simplexes of $P$ which have vertices lying in Q. Star $Q$ is the union of all closed simplexes of $P$ which meet $Q$. $Q$ is said to be full in $P$ if it contains each simplex of $P$, all of whose vertices lie in $Q$.

Henceforth all simplexes will be considered closed. If $\sigma$ is a simplex of $P$, by $\sigma^{*}$ we mean the union of all simplexes of $P$ which contain $\sigma$, or equivalently $\sigma^{*}=\sigma \circ \mathrm{Lk} \sigma$-where $\operatorname{Lk} \sigma$ is the link of $\sigma$ in $P$; $\sigma^{*}$ is called the cluster of $\sigma . \dot{\sigma}$ is the union of all proper faces of $\sigma ; \sigma^{b}=\sigma-\dot{\sigma}$. Finally $\beta(\sigma)$ will be the barycenter of $\sigma$.

THEOREM 6. Let $K$ be a full finite subpolyhedron of a polyhedral $n$ manifold $M$. Then $K$ is cellular in $M$ if and only if its stellar neighborhood is homeomorphic to $E^{n}$.

Proof. (a) Assume $K$ is cellular. Let $V$ be the stellar neighborhood of $K, B=\operatorname{Bd} V$ and $N=\operatorname{Star} K=\bar{V}$. Clearly $B \neq \varnothing$. Let $X$ be the set of all midpoints of straight segments joining points of $B$ to points of $K$. We shall show that the decomposition space of $V$ with $K$ identified to a point is homeomorphic to the open cone over $X$. For since $K$ is full, each simplex of $N$ can be represented as a join $\sigma \circ \tau, \sigma \subseteq K$, $\tau \subseteq B$. When $K$ is identified to a point, $\sigma \circ \tau-\sigma$ becomes simply the open cone over the set of all midpoints of segments from $\tau$ to $\sigma$; the vertex of the cone is the image of $K$ in the decomposition space. Since 
such a representation holds for each maximal simplex in $N$, the assertion is easily established.

Now let $C$ be a topological $n$-cell lying in $V$ with $K \subseteq$ Int $C$. By Theorem 1 of [1] there is a map $f$ of $V$ on itself fixed outside of $C$ such that $f(K)$ is a point and $f \mid V-K$ is one-to-one. Hence the existence of the map $f$ shows that $V$ is homeomorphic to the open cone over $X$, with vertex $f(K)$. By Theorem 4 and its corollary, $V \approx E^{n}$.

(b) Now suppose $V \approx E^{n}$. Since $K$ is full we can conclude from the argument in part (a) that $V$ can be represented as an open mapping cylinder over $K$ (from the space $X$ ) using the segments from $B$ to $K$ minus their endpoints in $B$. Since $K$ is compact there is a topological $n$-cell $C_{1}$ in $V$ so that $K \subseteq$ Int $C_{1}$. Now let each segment be linearly parametrized from 0 to 1 with 0 the parameter of the endpoint in $K$. There is an $\epsilon_{1}, 0<\epsilon_{1}<1 / 2$, so that on each segment the subsegment from 0 to $\epsilon_{1}$ lies in Int $C_{1}$. Hence let $h_{1}$ be a homeomorphism of $V$ into Int $C_{1}$, fixed on $K$, which maps each ray from 0 to 1 linearly onto its subray from 0 to $\epsilon_{1}$. Thus there is a topological $n$-cell $C_{2}$ in Int $C_{1}$ with $K \subseteq$ Int $C_{2}$. The process may be continued by induction to define a sequence $\left(C_{n}\right)$ of $n$-cells having the properties that $\bigcap_{n} C_{n}=K$ and $C_{n+1} \subseteq$ Int $C_{n}$.

CoRollary. Let $K$ be a finite subpolyhedron of a polyhedral $n$-manifold $M$. Then $K$ is cellular if and only if its first barycentric stellar neighborhood in $M$ is homeomorphic to $E^{n}$.

Proof. The first barycentric subdivision of $K$ is full in the corresponding subdivision of $M$ by Lemma 9.4, p. 71 of [2].

It may be noted that Theorem 6 generalizes Theorem 5 since vertices are clearly cellular.

Theorem 7. Let $\sigma$ be a simplex in a polyhedral $n$-manifold $M$. Then Int $\sigma^{*}$ is homeomorphic to $E^{n}$.

Proof (Added in proof). Adapting the notation of Alexander in [8] (our manifolds may be noncompact) let $M=\sigma \circ \mathrm{Lk} \sigma+R$ and consider the simple subdivision $M \rightarrow \beta(\sigma) \circ(\dot{\sigma} \circ \mathrm{Lk} \sigma)+R$. By Theorem $5, E^{n}$ is homeomorphic to $\operatorname{Int}(\beta(\sigma) \circ(\dot{\sigma} \circ \operatorname{Lk} \sigma))=\operatorname{Int} \sigma^{*}$. This method also tells us that, by Theorem $4, S(\dot{\sigma} \circ \mathrm{Lk} \sigma) \approx S^{n}$. Hence we have the

Corollary (Added in proof). If $\sigma$ is a $k$-dimensional simplex in a triangulated $n$-manifold then $S^{k} \circ \mathrm{Lk} \sigma \approx S^{n}$.

Proof (Added in proof). For $S(\dot{\sigma} \circ \mathrm{Lk} \sigma)=S(\dot{\sigma}) \circ \mathrm{Lk} \sigma$. 
TheOREM 8. Let $M$ be an $n$-manifold with triangulation $T$. Let $\sigma$ be a simplex of the first barycentric subdivision $T^{\prime}$ of $T$. Then $\sigma$ is cellular in $M$.

Proof. Let $\tau$ be the lowest dimensional simplex of $T$ for which $\beta(\tau)$ is a vertex of $\sigma$. Obviously $\tau^{b} \subseteq$ Int $\sigma^{*}$. We shall show that Star $\sigma \subseteq \tau^{*}$, Star $\sigma$ formed with respect to $T^{\prime}$ and $\tau^{*}$, with respect to $T$. For any other vertex $\beta\left(\tau^{\prime}\right)$ of $\sigma, \tau^{\prime} \supseteq \tau$; so if $\tau^{n}$ is an $n$-dimensional simplex containing $\beta\left(\tau^{\prime}\right)$, we have $\tau^{n} \supseteq \tau$ and hence $\tau^{n} \subseteq \tau^{*}$. It now may easily be seen that $\sigma \subseteq \operatorname{Int} \tau^{*}$.

Again consider the simple subdivision $M=\tau \circ \mathrm{Lk} \tau+R$ $\rightarrow \beta(\tau) \circ(\dot{\tau} \circ \mathrm{Lk} \tau)+R$. We can see that $\sigma$ is even a subcone, thus a compact starlike set in $\operatorname{Int}(\beta(\tau) \circ(\dot{\tau} \circ \operatorname{Lk} \tau))=\operatorname{Int} \tau^{*}$. Since Int $\tau^{*}$ $\approx E^{n}$ and by Theorem $2 \sigma$ is pointlike in Int $\tau^{*}$, it follows by Theorem 3 of [1] that $\sigma$ is cellular.

COROLlaRY. The stellar neighborhood of $\sigma$ with respect to $T^{\prime}$ is homeomorphic to $E^{n}$.

Proof. $\sigma$ is full in $T^{\prime}$.

Theorem 9 (Added in proof). Let $M$ be an n-manifold with triangulation $T$. If $\sigma$ is a simplex of the second barycentric subdivision $T^{\prime \prime}$ of $T$ then $\sigma^{*}$ is cellular in $M$.

Proof (Added in proof). $T^{\prime}$ will denote the first barycentric subdivision of $T$. Suppose $\sigma$ is a simplex of $T^{\prime \prime}$. Let $\tau$ be the lowest dimensional simplex of $T^{\prime}$ whose barycenter $\beta(\tau)$ is a vertex of $\sigma$. As before, it follows that $\sigma^{*} \subseteq \tau^{*}$. It is also fairly easy to see that each vertex of $\tau$ lies in $\sigma^{*}$.

Now let $v$ be the minimal simplex of $T$ whose barycenter $\beta(v)$ is a vertex of $\tau$. We may conclude that $v$ is the minimal simplex of $T$ whose barycenter lies in $\sigma^{*}$. For if $v_{1} \in T$ and $\beta\left(v_{1}\right) \in \sigma^{*}$, denote by $\sigma_{1}$ the simplex of $T^{\prime \prime}$ which contains $\sigma$ and $\beta\left(v_{1}\right)$. Since $\sigma_{1}$ must be $n$ dimensional let $\tau_{1}$ be the $n$-simplex of $T^{\prime}$ containing $\sigma_{1}$. Inasmuch as $\sigma_{1} \supseteq \sigma, \tau_{1} \supseteq \tau$ and $\beta(\tau) \in \sigma$, it may be seen that $\beta\left(v_{1}\right) \in \tau$. This proves that $v \subseteq v_{1}$.

Consider the simple subdivision $M=v \circ \mathrm{Lk} v+R \rightarrow \beta(v) \circ P+R$, where $P=\dot{v} \circ \mathrm{Lk} v$. Evidently $\sigma^{*} \subseteq \beta(v) \circ P$; we shall prove that $\sigma^{*} \subseteq \operatorname{Int} \beta(v) \circ P$, the latter, of course, being a homeomorph of $E^{n}$. Firstly, it may be established without too much difficulty, by a straightforward induction on $n$, that if $\tau^{n} \subseteq \beta(v)^{*}$ with respect to $T^{\prime}$ then $\tau^{n} \cap P \subseteq \dot{v}$. Now suppose $\sigma^{n} \in T^{\prime \prime}$ so that $\sigma^{n} \supseteq \sigma$ and $\sigma^{n} \cap \dot{v} \neq \varnothing$. Let $\tau^{n} \in T^{\prime}$ with $\sigma^{n} \subseteq \tau^{n}$ and $\tau_{1}=\tau^{n} \cap \dot{v}$. Then $\sigma^{n}$ contains $\beta\left(\tau_{0}\right)$ for 
some face $\tau_{0}$ of $\tau_{1}$; this in turn necessitates that $\sigma^{n}$ contains a vertex $v$ of $\tau_{0}$. The vertex $v$ must be the barycenter of a face $v_{0}$ of $\dot{v}$. Since $v \in \sigma^{*}$, this contradicts the minimality condition on $v$ proved above.

Finally we show that $\sigma^{*}$ is starlike in $\beta(v) \circ P$. It will then be obvious from our previous arguments that $\sigma^{*}$ is cellular in $M$. Let $\tau^{n}$ be any $n$-simplex of $T^{\prime}$ which contains $\sigma$. By elementary analytic geometry it may be verified that $\sigma^{*} \cap \tau^{n}$ is convex in $\tau^{n}$. (See for example formula (2) on p. 62 of [2].) This completes the proof of the theorem.

\section{REFERENCES}

1. M. Brown, $A$ proof of the generalized Schoenflies theorem, Bull. Amer. Math. Soc. 66 (1960), 74-76.

2. S. Eilenberg and N. Steenrod, Foundations of algebraic topology, Princeton Univ. Press, Princeton, N. J., 1952.

3. B. Mazur, On embeddings of spheres, Bull. Amer. Math. Soc. 65 (1959), 59-65.

4. - On embeddings of spheres, Acta Math. 105 (1961), 1-17.

5. E. E. Moise, Affine structures in 3-manifolds. VI, Ann. of Math. (2) 58 (1953), 107.

6. M. Morse, A reduction of the Schoenflies extension problem, Bull. Amer. Math. Soc. 66 (1960), 113-115.

7. R. Rosen, $A$ weak form of the star conjecture for manifolds, Abstract 570-28, Notices Amer. Math. Soc. 7 (1960), 380.

8. J. W. Alexander, The combinatorial theory of complexes, Ann. of Math. (2) 31 (1930), 292-320.

UNIVERSITY OF MichigaN 